Check for updates

Cite this: Chem. Commun., 2021,

57, 2887

Received 10th December 2020,

Accepted 4th February 2021

DOI: $10.1039 / \mathrm{d} 0 \mathrm{cc} 08025 \mathrm{j}$

rsc.li/chemcomm

\section{Heteroternary cucurbit[8]uril complexes as supramolecular scaffolds for self-assembled bifunctional photoredoxcatalysts +}

\author{
Fabian Lutz, ${ }^{a}$ Nerea Lorenzo-Parodi, ${ }^{\mathrm{b}}$ Torsten C. Schmidt (D) ${ }^{\mathrm{b}}$ and \\ Jochen Niemeyer (iD *a
}

The self-assembly of bifunctional photoredoxcatalysts is reported. A series of photosensitizers and water-reducing catalysts were functionalized with viologen- and naphthol-units, respectively. Subsequent formation of the heteroternary cucurbit[8]uril-viologennaphthol complexes was used for the constitution of bifunctional photoredoxcatalysts for hydrogen generation.

Since the industrial revolution, mankind has steadily increased its power demands. Especially in the last hundred years, power consumption has risen drastically due to population growth and rising living standards. ${ }^{1}$ As of today, most energy sources are based on limited fossil fuels like oil, gas and coal and represent a main factor in global warming. ${ }^{2}$ One possible alternative is solar energy, harnessed by photovoltaics, by thermal collectors or via photocatalysis. Especially the photocatalytic production of dihydrogen is attractive, since $\mathrm{H}_{2}$ can be stored and subsequently be transformed into electrical energy in fuel cells in an emission-free fashion. ${ }^{3}$

Photocatalytic hydrogen production can be effected by bifunctional photocatalysts consisting of a photosensitizer (PS) and a water reducing catalyst (WRC). Starting with research by Lehn and Sauvage in 1977, the development of such bifunctional (often bimetallic) catalysts has been met with high interest. ${ }^{4}$

Initially, heterobimetallic photocatalysts were generated by dissolving two suitable metal complexes in aqueous or organic solutions. ${ }^{5}$ However, the efficiency of the intermolecular electron transfer between PS and WRC is distant-dependent, so that simple dissolution of both components renders such systems inefficient at low concentrations. ${ }^{6}$ This can be circumvented by

\footnotetext{
${ }^{a}$ Faculty of Chemistry, Organic Chemistry and Center for Nanointegration Duisburg-Essen (CENIDE), University of Duisburg-Essen, Universitätsstrasse. 7, Essen 45141, Germany. E-mail: jochen.niemeyer@uni-due.de

${ }^{b}$ Instrumental Analytical Chemistry and Centre for Water and Environmental

Research (ZWU), Universitätsstrasse 5, Essen 45141, Germany

$\dagger$ In memory of Prof. Carsten Schmuck.

\# Electronic supplementary information (ESI) available. See DOI: 10.1039/ d0cc08025j
}

integrating both metal centers into a single molecule, enabling intramolecular electron transfer. ${ }^{7}$ Unfortunately, the exact influence of the ligands on electron transfer is hard to predict ${ }^{8}$ and many bimetallic complexes are sparingly soluble in pure water. $^{4 b, 9}$

Supramolecular self-assembly is a powerful tool to induce spatial proximity in a bifunctional catalyst, ${ }^{10}$ which may also facilitate the electron transfer between both subunits of a photocatalyst. Based on the use of hydrophobic interactions, supramolecular self-assembly can also be applied in aqueous solvents. ${ }^{11}$ In this context, König and coworkers employed functionalized vesicles together with alkylated PSs and WRCs to achieve a 2D-confinement of the photocatalyst components. ${ }^{12}$ The alkyl-chains are anchored onto the vesicle surface, leading to a strongly increased local concentration. Although dynamic in nature, this approach enhances photocatalytic efficiency.

We envisaged that supramolecular chemistry can also be used to achieve a more defined spatial preorganisation of two subcomponents. Especially the use of macrocyclic hosts, such as cyclodextrins, calixarenes or cucurbiturils, seems promising in this regard, since these host-molecules can bind hydrophobic guests in their inner cavity with high association constants. ${ }^{13}$ In this work, we employ cucurbit[8]uril (CB8), which possesses a nonpolar cavity surrounded on both sides by carbonyl groups (Fig. 1B). CB8 itself is almost completely insoluble in water, but can be solubilized by formation of the corresponding host-guest complexes. CB8 can bind up to two guests within its cavity at once, leading to binary $2: 1$ or heteroternary $1: 1: 1$ complexes. For the formation of 1:1:1 complexes, dialkylviologen and 2-naphthol are suitable guest molecules. Although 2-naphthol itself does not bind within CB8, it does bind to the preformed CB8-viologen complex, leading exclusively to the 1:1:1 heteroternary complex with association constants of $1.1 \times 10^{5} \mathrm{M}^{-1}$ for the first and $5.9 \times 10^{5} \mathrm{M}^{-1}$ for the second step (Fig. 1A). ${ }^{14}$ This is due to the formation of a charge-transfer complex between the electron poor viologen and the electron rich naphthol unit. ${ }^{15}$ 


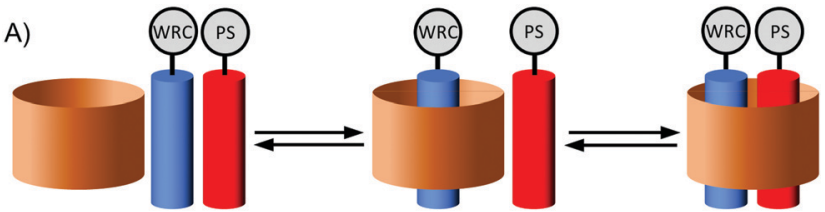

B)

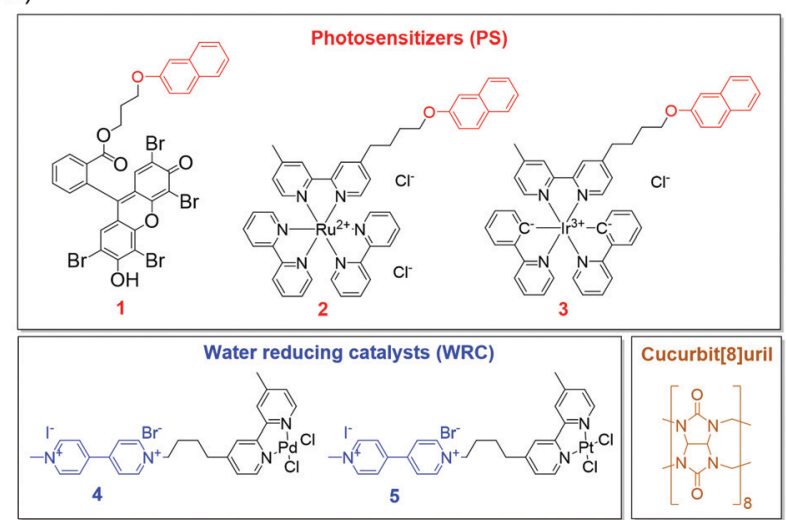

Fig. 1 (A) Concept for the self-assembly of photocatalysts for hydrogengeneration based on heteroternary 1:1:1 host-guest complexes of CB8 (B) Molecular structures of the functionalized photosensitizers, functionalized water reducing catalysts and cucurbit[8]uril.

With this in mind, we aimed for the functionalization of 2-naphthol and viologen with photosensitizers and waterreducing-catalysts, respectively. In presence of $\mathbf{C B 8}$, a heteroternary PS-WRC-CB8 complex with controlled stoichiometry should be formed, leading to a spatial proximity of the catalyst subcomponents and thus enhancing photocatalytic efficiency. Different combinations of PS and WRC were investigated with regard to their ability for hydrogen generation, leading to the identification of an Ir/Pt pair as the most efficient photocatalyst.

First, we synthesized the functionalized PSs and WRCs. As PSs, trisbipyridine-ruthenium(II) and bipyridine-bis(2-phenylpyridine)-iridium(III) complexes were used as well as a metal-free eosine Y derivative. WRCs are based on bipyridine-palladium and -platinum complexes. As viologen is highly water soluble it was coupled to the uncharged Pd- and Pt-complexes, whereas the nonpolar naphthol-unit was conjugated with the water soluble $\mathrm{Ru}$ - and Ir-complexes (also via the bipyridine ligand) as well as the eosin Y derivative (via the carboxylic acid functionality). This resulted in three functionalized PSs 1/2/3 and two WRCs $4 / 5$ (Fig. 1B). All compounds were fully characterized by standard techniques (see ESI $\ddagger$ ).

Subsequently, we generated the heteroternary complexes in water containing $15 \%(\mathrm{v} / \mathrm{v})$ triethanolamine (TEOA), which acts as a sacrificial electron donor (for the effect of different TEOAconcentrations on the $\mathrm{H}_{2}$-production see Table S8, ESI ). CB8, which is insoluble in this solvent mixture, could be dissolved in the presence of both guest molecules (presence of PS or WRC alone was not sufficient), indirectly proving the successful formation of the heteroternary $1: 1: 1$ complexes. The complex formation could also be followed by ${ }^{1} \mathrm{H}-\mathrm{NMR}$ spectroscopy: in the $\mathbf{3}+\mathbf{1 0}+\mathbf{C B 8}$ mixture (10: viologen-bipyridine ligand, Fig. S2, ESI $\ddagger$ ), the signals for CB8 were shifted to 6.08/5.78 ppm ( $c f .6 .18$ /
5.84 ppm for free CB8) and the signals for the viologen-protons were severely broadened, which is characteristic for formation of the charge-transfer complex (Fig. S19, ESI $\$$ ). ${ }^{16}$ Yet, the CB8-unit did not show a reduced symmetry upon complex formation, which we attribute to a fast guest exchange on the NMR time scale, as found by other groups. ${ }^{17}$ Furthermore, the samples showed a change in color from yellow to purple due to formation of the charge-transfer complex. ${ }^{16}$ In the UV/Vis spectra, the charge transfer band could not be observed directly due to overlap with the absorption bands of the coloured PSs. ${ }^{18}$ However, the UV/Vis absorption and the fluorescence of the heteroternary complexes are decreased in comparison to the mixture of PSs and WRCs alone. For example, the $\mathbf{3}+\mathbf{5}+\mathbf{C B 8}$ mixture shows a $12 \%$ decrease for the UV/Vis absorption at $320 \mathrm{~nm}$ and even a $36 \%$ decrease in the fluorescence intensity of the iridium complex at $575 \mathrm{~nm}$ (Fig. S35 and S36, ESI $\$$ ). The decrease in fluorescence indicates a non-emissive deactivation of the excited state of the PS, e.g. by PET to the WRC. ${ }^{7 b, 8 a}$ This hints at a spatial proximity of the PS and WRC, which in conjunction with the data from NMR, optical spectroscopy and solubility indicate the formation of the desired CB8-based host-guest complexes.

Next, the application of the bifunctional supramolecular catalysts in hydrogen evolution was tested. Irradiation experiments were conducted at a minimum concentration of $0.25 \mathrm{mM}$ for each component, which should allow sufficient complex formation based on the reported association constants $\left(>10^{5} \mathrm{M}^{-1}\right)$. To ensure full homogeneity, the mixture was sonicated in an ultrasonic bath for 15 minutes before being placed in a lab shaker for 10 hours in the dark at $60{ }^{\circ} \mathrm{C}$. The temperature was chosen to accommodate for the low solubility of the water reducing catalysts. After flushing carefully with argon, irradiation was carried out at $60{ }^{\circ} \mathrm{C}$ for 11 hours with LEDs of suitable wavelength for each PS (528/470/ $365 \mathrm{~nm}$ for $\mathbf{1 / 2} / \mathbf{3}$ ). The amount of generated hydrogen was determined by headspace gas chromatography (based on external calibration, Table S1, ESI $\ddagger$ ) and is reported as turn-over-number (TON), normalized to a concentration of the least concentrated component (always $0.25 \mathrm{mM}$ ).

First, all six possible combinations of PSs 1/2/3 and WRCs 4/ 5 with CB8 were tested in a 1:1:1 stoichiometry $0.25 \mathrm{mM}$ each). For palladium complex 4, we did not observe any relevant generation of hydrogen in combination with either of the photosensitizers (Tables S2, S3 and S5, ESI $\$$ ). In contrast, platinum complex $\mathbf{5}$ was suitable as the WRC in this approach: in combination with eosin-based photosensitizer $\mathbf{1}$, we observed $1.31 \pm 0.02$ TONs (Table S2, ESI $\$$ ). However, an identical amount of hydrogen was produced in the absence of CB8. We attribute this to the low solubility of 1: even in the presence of $\mathbf{C B 8}$, which allowed complete dissolution of all components for $2 / 3$ in combination with 5 (vide infra), a red precipitate was observed in case of $\mathbf{1}$.

To our delight, the combination of PS 2 and WRC 5 showed a positive effect of CB8 in photocatalytic hydrogen generation (Table S3, ESI ). For the 1:1:1 stoichiometry, we observed only $0.50 \pm 0.06$ TONs in the absence of CB8, while addition of CB8 gave increased TONs, albeit with low reproducibility 


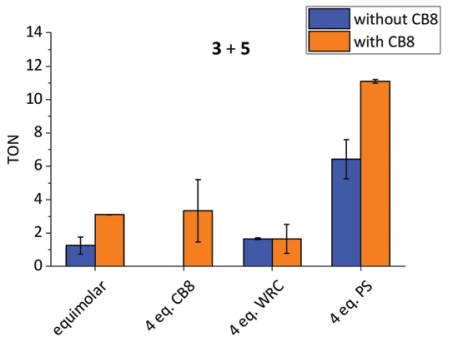

Fig. 2 Amount of hydrogen generated by combination of PS 3 and WRC 5 at different stoichiometries at concentrations of $0.25 \mathrm{mmol} \mathrm{l}^{-1}$ or $1.0 \mathrm{mmol} \mathrm{l}^{-1}$ for each component in a mixture of water/triethanolamine (85/15). Turnover numbers are normalized for a concentration of $0.25 \mathrm{mM}$.

(1.19 \pm 1.26 TONs). Further experiments were executed in which one compound was added in excess (4 equivalents, i.e. $1 \mathrm{mM}$ concentration), showing a drastic increase in photocatalytic activity in presence of four equivalents of WRC 5, although the effect of the $\mathbf{C B 8}$ is rather low $(9.38 \pm 0.75 / 11.8 \pm 0.45$ TONs in absence/presence of CB8, i.e. $+26 \%$, Table S4, ESI $\$)$.

Finally, we investigated the use of the Ir-based PS 3 in combination with WRC 5 (Fig. 2 and Tables S5, S6, ESI ). In comparison to the $\mathbf{2}+\mathbf{5}$ combination, significantly higher TONs were observed when used in equimolar ratio $(1.26 \pm 0.51 / 3.11 \pm$ 0.01 TONs for $3+5$ in the absence/presence of CB8, $c f .0 .50 \pm$ $0.06 / 1.19 \pm 1.26$ for $2+5$ ). More importantly, the $1: 1: 1$ ternary mixture now gives reproducible results and the presence of CB8 leads to strong increase in hydrogen evolution $(+147 \%)$. Once again, we checked the influence of adding each component in excess (4 equiv.). Here, the addition of excess WRC did not lead to an increased activity $(1.65 \pm 0.87$ TONs for 4 equiv. of $5, c f$. $3.11 \pm 0.01$ TONs for the $1: 1: 1$ system). In contrast, increasing the concentration of PS 3 led to increased TONs, indicating that the PS deteriorates more quickly than the WRC in this case. Without CB8, the mixture of $3(1 \mathrm{mM})$ with $5(0.25 \mathrm{mM})$ gives rise to $6.42 \pm 1.17$ TONs, while complexation by CB8 leads to an increased activity of $11.1 \pm 0.11$ TONs $(+73 \%)$.

Next, a series of control experiments was performed in order to check the viability of our proposed mechanism, namely the improved photoinduced electron transfer by spatial proximity of the reaction partners in the $\mathbf{3}+\mathbf{5}+\mathbf{C B 8}$ host guest complex (Fig. 3 and Table S7, ESI $\$$ ). To demonstrate the photocatalytic character of the hydrogen generation, the reaction was carried out without irradiation and indeed no hydrogen generation was observed. Then, we checked the possible formation of catalytically active colloidal Pt-nanoparticles, by addition of elemental mercury, which however did not change the catalytic activity $(3.11 \pm 0.01 / 3.72 \pm 0.50$ TONs in absence/presence of $\mathrm{Hg}) .{ }^{19}$ Next, the importance of each component in the heteroternary complex was tested by substituting PS 3, WRC 5 or both by unfunctionalized 2-naphthol (naph) or ethylviologen (EV) moieties. The metal-free combination (naph + EV + CB8) showed hardly any hydrogen generation and the heteroternary complexes $\mathbf{3}+$ EV $+\mathbf{C B 8}$ and naph $+\mathbf{5}+\mathbf{C B 8}$ only gave a reduced catalytic activity $(0.72 \pm 0.60 / 1.16 \pm 0.34$ TONs, $c f .3 .11 \pm 0.01$ TONs for the $\mathbf{3}+\mathbf{5}+\mathbf{C B 8}$ mixture). Also, we investigated if the covalent

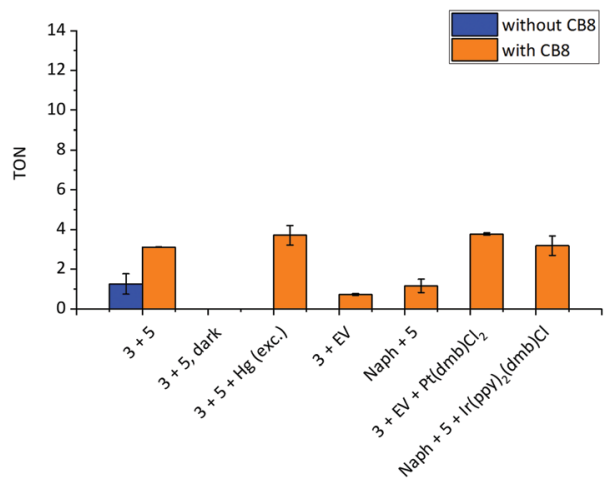

Fig. 3 Amount of hydrogen generated by combinations of PS 3 and WRC 5 and different mechanistic control reactions at concentrations of $0.25 \mathrm{mmol} \mathrm{l}^{-1}$ for each component in a mixture of $15: 85$ triethanolamine : water. Turnover numbers are normalized for a concentration of $0.25 \mathrm{mM}$.

binding of the metal to the anchoring unit is necessary by using the mixtures of $\mathbf{3}+\mathrm{EV}+\mathbf{C B 8}$ with $\mathrm{Pt}(\mathrm{dmb}) \mathrm{Cl}_{2}$ or Naph $+\mathbf{5}+\mathbf{C B 8}$ with $\operatorname{Ir}(\mathrm{ppy})_{2}(\mathrm{dmb}) \mathrm{Cl}$, respectively (ppy: 2-phenyl-pyridyl, dmb: $4,4^{\prime}$-dimethyl-2,2'-bipyridine). Surprisingly, this led to a catalytic activity $(3.77 \pm 0.06 / 3.19 \pm 0.49$ TONs) comparable to the system consisting of $3+5+\mathbf{C B 8},(3.11 \pm 0.01$ TONs). Although other explanations might be possible, we believe that this might indicate an anti-orientation of the metal centers in the $\mathbf{3}+\mathbf{5}+$ CB8 system, spatially separating photosensitizer and water reducing catalyst, thus obliviating the effect of the covalent linking to the anchoring unit.

Thus, we envisaged the application of the bis-Pt viologen derivative 6 (Fig. 4, for the synthesis of 6 see the ESI $\$$ ), which would ensure that one Pt-center is always in close proximity to the Ir-center in the $\mathbf{3}+\mathbf{6}+\mathbf{C B 8}$ complex. ${ }^{20}$ Force-field calculations show that the bis-Pt complex is able to thread the CB8 macrocycle despite the two bipyridine- $\mathrm{PtCl}_{2}$ termini (Fig. S37 and S38, ESI + ). The formation of the heteroternary complexes is also supported by decreases in absorbance (37\% decrease at $320 \mathrm{~nm}$ ) and fluorescence ( $50 \%$ decrease at $575 \mathrm{~nm}$ ) upon addition of CB8 to the $3+6$ mixture (Fig. S35 and S36, ESI $\$$ ).

In the catalytic application, we used the optimized stoichiometry (4 equiv. PS, 1 equiv. WRC, 1 equiv. CB8), to test the

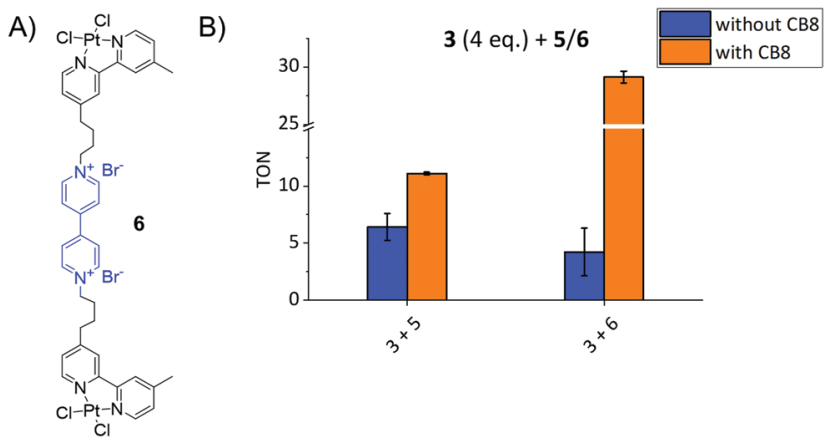

Fig. 4 (A) Structure of bisplatinated complex 6. (B) Comparison between the system of $\mathbf{3}, \mathbf{5}$, and CB8 with an excess of PS $\mathbf{3}$, and the use of bisplatinated complex 6 at concentrations of $0.25 \mathrm{mmol} \mathrm{l}^{-1}$ or $1.0 \mathrm{mmol} \mathrm{l}^{-1}$ for each component in a mixture of water/triethanolamine (85/15). 
Table 1 Summary of the photocatalytic activity of different combinations of PS and WRC, with and without CB8 (as shown in Fig. 2-4)

\begin{tabular}{|c|c|c|}
\hline \multirow[b]{2}{*}{ Experiment } & \multicolumn{2}{|l|}{ TON } \\
\hline & Without CB8 & With CB8 \\
\hline $3+5$ & $1.26 \pm 0.51$ & $3.11 \pm 0.01$ \\
\hline $3+5$ (4 equiv. $\mathbf{C B 8})$ & - & $3.34 \pm 1.88$ \\
\hline $3+4$ equiv. 5 & $1.66 \pm 0.07$ & $1.65 \pm 0.87$ \\
\hline 4 equiv. $3+5$ & $6.43 \pm 1.17$ & $11.1 \pm 0.11$ \\
\hline $3+5$ (dark) & - & 0 \\
\hline $3+5+\mathrm{Hg}$ & - & $3.72 \pm 0.50$ \\
\hline $3+E V$ & - & $0.72 \pm 0.05$ \\
\hline $\mathrm{Naph}+\mathbf{5}$ & - & $1.16 \pm 0.34$ \\
\hline $3+\mathrm{EV}+$ Ptdmb & - & $3.77 \pm 0.06$ \\
\hline $\mathrm{Naph}+\mathbf{5}+\mathrm{Irdmb}$ & - & $3.19 \pm 0.49$ \\
\hline 4 equiv. $3+6$ & $4.23 \pm 2.09$ & $29.1 \pm 0.53$ \\
\hline
\end{tabular}

substitution of the mono-Pt complex 5 by the bis-Pt derivative 6 (Fig. 4 and Table S6, ESI‡). In absence of CB8, no change was observed $(4.23 \pm 2.09$ TONs for $3+6, c f .6 .43 \pm 1.17$ TONs for $3+5$ ), which also indicates that the effective increase in total Pt-concentration has no effect (as expected based on the stoichiometry experiments for the $\mathbf{3}+\mathbf{5}$ mixture, see Table S6, ESI $\$$. However, in the presence of CB8, a significant increase in hydrogen-production was observed when using the bis-Pt system $6(29.1 \pm 0.53$ TONs for $3+\mathbf{6}+\mathbf{C B 8}, c f .11 .1 \pm 0.11$ TONs for $3+5+$ CB8). One possible explanation for this substantial increase in hydrogen production $(+263 \%)$ is that the spatial proximity of the metal-centers is improved by ruling out the anti-orientation (Table 1).

In summary, we have reported a supramolecular concept for the photocatalytic generation of hydrogen from aqueous solutions. Formation of heteroternary 1:1:1 complexes of CB8 with naphthol-functionalized PSs and viologen-functionalized WRCs allowed for an efficient screening of different PS/WRC combinations, leading to the identification of the Ir/Pt pair as the best candidate. The initial hit $(3+5+\mathrm{CB}, 3.11 \pm 0.01$ TONs $)$ was systematically investigated and optimized by variation of stoichiometry and a series of control experiments, finally leading to the $\mathbf{3}+\mathbf{6}+\mathbf{C B 8}$ system with a largely increased activity $(29.1 \pm 0.53$ TONs with 4 equiv. 3 , i.e. $+935 \%$ vs. $\mathbf{3}+\mathbf{5}+\mathbf{C B})$. Thus, CB8-based supramolecular self-assembly can be an efficient tool for the formation of bifunctional catalysts in aqueous solution and we are currently expanding this concept towards combined transition-metal and organocatalysts.

Funding from MERCUR (AN-2016-007) and the Fonds der Chemischen Industrie (Liebig-Fellowship) is gratefully acknowledged. We thank Prof. Burkhard König and Dr. Andreas Brunschweiger for their support and Jun.-Prof. Jens Voskuhl for discussions.

\section{Conflicts of interest}

There are no conflicts to declare.

\section{Notes and references}

1 British Petroleum Company, BP Statistical Review of World Energy, British Petroleum Co, London, 2019.
2 M. Höök and X. Tang, Energy Policy, 2013, 52, 797-809.

3 C.-H. Liao, C.-W. Huang and J. C. Wu, Catalysts, 2012, 2, 490-516.

4 (a) J.-M. Lehn and J.-P. Sauvage, Nouv. J. Chim., 1977, 1, 449-451; (b) J. Corredor, M. J. Rivero, C. M. Rangel, F. Gloaguen and I. Ortiz, J. Chem. Technol. Biotechnol., 2019, 94, 3049-3063.

5 (a) T. Stoll, C. E. Castillo, M. Kayanuma, M. Sandroni, C. Daniel, F. Odobel, J. Fortage and M.-N. Collomb, Coord. Chem. Rev., 2015, 304-305, 20-37; (b) A. Zarkadoulas, E. Koutsouri, C. Kefalidi and C. A. Mitsopoulou, Coord. Chem. Rev., 2015, 304-305, 55-72; (c) A. Inagaki and M. Akita, Coord. Chem. Rev., 2010, 254, 1220-1239.

6 D. M. Arias-Rotondo and J. K. McCusker, Chem. Soc. Rev., 2016, 45, 5803-5820.

7 (a) S. Rau, B. Schäfer, D. Gleich, E. Anders, M. Rudolph, M. Friedrich, H. Görls, W. Henry and J. G. Vos, Angew. Chem., Int. Ed., 2006, 45, 6215-6218; (b) J. K. White and K. J. Brewer, Chem. Commun., 2015, 51, 16123-16126; (c) R. Zhou, G. F. Manbeck, D. G. Wimer and K. J. Brewer, Chem. Commun., 2015, 51, 12966-12969; (d) M. G. Pfeffer, B. Schäfer, G. Smolentsev, J. Uhlig, E. Nazarenko, J. Guthmuller, C. Kuhnt, M. Wächtler, B. Dietzek, V. Sundström and S. Rau, Angew. Chem., Int. Ed., 2015, 54, 5044-5048; (e) D. Gust, Faraday Discuss., 2015, 185, 9-35; $(f)$ M. G. Pfeffer, T. Kowacs, M. Wächtler, J. Guthmuller, B. Dietzek, J. G. Vos and S. Rau, Angew. Chem., Int. Ed., 2015, 54, 6627-6631.

8 (a) G. Singh Bindra, M. Schulz, A. Paul, S. Soman, R. Groarke, J. Inglis, M. T. Pryce, W. R. Browne, S. Rau, B. J. Maclean and J. G. Vos, Dalton Trans., 2011, 40, 10812-10814; (b) S. Tschierlei, M. Karnahl, M. Presselt, B. Dietzek, J. Guthmuller, L. González, M. Schmitt, S. Rau and J. Popp, Angew. Chem., Int. Ed., 2010, 49, 3981-3984.

9 Y. Halpin, M. T. Pryce, S. Rau, D. Dini and J. G. Vos, Dalton Trans., 2013, 42, 16243-16254.

10 (a) R. Mitra, H. Zhu, S. Grimme and J. Niemeyer, Angew. Chem., Int. Ed., 2017, 56, 11456-11459; (b) R. Mitra and J. Niemeyer, ChemCatChem, 2018, 10, 1221-1234; (c) D. Kauerhof and J. Niemeyer, ChemPlusChem, 2020, 85, 889-899; (d) N. Pairault, H. Zhu, D. Jansen, A. Huber, C. G. Daniliuc, S. Grimme and J. Niemeyer, Angew. Chem., Int. Ed., 2020, 59, 5102-5107.

11 Y. Kumar, B. Patil, A. Khaligh, S. E. Hadi, T. Uyar and D. Tuncel, ChemCatChem, 2019, 11, 2994-2999.

12 (a) S. Troppmann and B. König, Chem. - Eur. J., 2014, 20, 14570-14574; (b) S. Troppmann, E. Brandes, H. Motschmann, F. Li, M. Wang, L. Sun and B. König, Eur. J. Inorg. Chem., 2016, 554-560; (c) A. Králík, M. Hansen and B. König, RSC Adv., 2016, 6, 5739-5744.

13 K. I. Assaf and W. M. Nau, Chem. Soc. Rev., 2015, 44, 394-418.

14 U. Rauwald, F. Biedermann, S. Deroo, C. V. Robinson and O. A. Scherman, J. Phys. Chem. B, 2010, 114, 8606-8615.

15 (a) H.-J. Kim, J. Heo, W. S. Jeon, E. Lee, J. Kim, S. Sakamoto, K. Yamaguchi and K. Kim, Angew. Chem., 2001, 113, 1574-1577; For the use of CB8 in photocatalysis, see: $(b)$ B. C. Pemberton, E. Kumarasamy, S. Jockusch, D. K. Srivastava and J. Sivaguru, Can. J. Chem., 2011, 89, 310-316; (c) B. C. Pemberton, N. Barooah, D. K. Srivatsava and J. Sivaguru, Chem. Commun., 2010, 46, 225-227; (d) B. C. Pemberton, R. K. Singh, A. C. Johnson, S. Jockusch, J. P. Da Silva, A. Ugrinov, N. J. Turro, D. K. Srivastava and J. Sivaguru, Chem. Commun., 2011, 47, 6323-6325; (e) B. C. Pemberton, R. Raghunathan, S. Volla and J. Sivaguru, Chem. - Eur. J., 2012, 18, 12178-12190.

16 D. Zou, S. Andersson, R. Zhang, S. Sun, B. Åkermark and L. Sun, J. Org. Chem., 2008, 73, 3775-3783.

17 (a) H. Chen, S. Hou and Y. Tan, Supramol. Chem., 2016, 28, 801-809; (b) M. E. Bush, N. D. Bouley and A. R. Urbach, J. Am. Chem. Soc., 2005, 127, 14511-14517.

18 F. Biedermann and O. A. Scherman, J. Phys. Chem. B, 2012, 116, 2842-2849.

19 (a) R. H. Crabtree, Chem. Rev., 2012, 112, 1536-1554; (b) G. M. Whitesides, M. Hackett, R. L. Brainard, J. P. P. M. Lavalleye, A. F. Sowinski, A. N. Izumi, S. S. Moore, D. W. Brown and E. M. Staudt, Organometallics, 1985, 4, 1819-1830.

20 As opposed to a through-space PET, it has also been reported that electron transfer can occur through the walls of the host-molecule: see: (a) F. S. Santos, E. Ramasamy, V. Ramamurthy and F. S. Rodembusch, Photochem. Photobiol. Sci., 2017, 16, 840-844; (b) C.-H. Chuang, M. Porel, R. Choudhury, C. Burda and V. Ramamurthy, J. Phys. Chem. B, 2018, 122, 328-337. 